\title{
Prevalence of Defaecatory Disorders in Morbidly Obese Patients Before and After Bariatric Surgery
}

\author{
Pierpaolo Sileri • Luana Franceschilli • Federica Cadeddu • Elisabetta De Luca • \\ Stefano D'Ugo • Valeria Tognoni • Ida Camperchioli • Domenico Benavoli • \\ Nicola Di Lorenzo • Achille L. Gaspari • Paolo Gentileschi
}

Received: 2 June 2011 / Accepted: 14 September 2011 /Published online: 24 September 2011

(C) 2011 The Society for Surgery of the Alimentary Tract

\begin{abstract}
Background The prevalence of obesity is increasing worldwide and has lately reached epidemic proportions in western countries. Several epidemiological studies have consistently shown that both overweight and obesity are important risk factors for the development of various functional defaecatory disorders (DDs), including faecal incontinence and constipation. However, data on their prevalence as well as effectiveness of bariatric surgery on their correction are scant. The primary objective of this study was to estimate the effect of morbid obesity on DDs in a cohort of patients listed for bariatric surgery. We also evaluated preliminary results of the effects of sleeve gastrectomy on these disorders.

Patients and methods A questionnaire-based study was proposed to morbidly obese patients having bariatric surgery. Data included demographics, past medical, surgical and obstetrics histories, as well as obesity related co-morbidities. Wexner Constipation Score (WCS) and the Faecal Incontinence Severity Index (FISI) questionnaires were used to evaluate constipation and incontinence. For the purpose of this study, we considered clinically relevant a WCS $\geq 5$ and a FISI score $\geq 10$. The same questionnaires were completed at 3 and 6 months follow-up after surgery.

Results A total of 139 patients accepted the study and 68 underwent sleeve gastrectomy and fully satisfied our inclusion criteria with a minimum follow-up of 6 months. Overall, mean body mass index (BMI) at listing was $47 \pm 7 \mathrm{~kg} / \mathrm{m}^{2}$ (range $35-67 \mathrm{~kg} / \mathrm{m}^{2}$ ). Mean WCS was $4.1 \pm 4$ (range 0-17), while mean FISI score (expressed as mean \pm standard deviation) was 9.5 \pm 9 (range $0-38$ ). Overall, $58.9 \%$ of the patients reported DDs according to the above-mentioned scores. Twenty-eight patients $(20 \%)$ had WCS $\geq 5$. Thirty-five patients $(25 \%)$ had a FISI $\geq 10$ while 19 patients $(13.7 \%)$ reported combined abnormal scores. Overall, DDs were more evident with the increase of obesity grade: Mean BMI decreased significantly from $47 \pm 7$ to $36 \pm 6$ and to $29 \pm 4 \mathrm{~kg} / \mathrm{m}^{2}$ respectively at 3 and 6 months after surgery $(p<0.0001)$. According to the BMI decrease, the mean WCS decreased from $3.7 \pm 3$ to $3.1 \pm 4$ and to $1.6 \pm 3$ respectively at 3 and 6 months $(p=0.02)$. Similarly, the FISI score decreased from $10 \pm 8$ to $3 \pm 4$ and to $1 \pm 2$ respectively at 3 and 6 months $(p=0.0001)$.

Conclusions Defaecatory disorders are common in morbidly obese patients. The risk of DDs increases with BMI. Bariatric surgery reduces DDs, mainly faecal incontinence, and these findings correlated with BMI reduction.
\end{abstract}

This study was presented at SSAT annual meeting 2011, Chicago IL.

P. Sileri $\cdot$ L. Franceschilli $\cdot$ F. Cadeddu $\cdot$ E. De Luca $\cdot$ S. D’Ugo $\cdot$

V. Tognoni · I. Camperchioli - D. Benavoli • N. Di Lorenzo •

A. L. Gaspari · P. Gentileschi

Department of Surgical Sciences, University of Rome Tor Vergata,

Rome, Italy

P. Sileri $(\bowtie)$

Policlinico Tor Vergata, UOC B,

Viale Oxford 81,

00133 Rome, Italy

e-mail: piersileri@yahoo.com
Keywords Morbid obesity - Faecal incontinence .

Defaecatory disorders · Sleeve gastrectomy $\cdot$ Constipation

\section{Introduction}

The prevalence of obesity is increasing worldwide and has lately reached epidemic proportions in western countries. ${ }^{1}$ In the USA, approximately $30 \%$ of adults ( $\geq 20$ years) are obese and $16 \%$ of children (6-19 years) are overweight. ${ }^{2}$ Morbid obesity is currently defined by having a body mass index 
(BMI, calculated dividing weight in kilogram per meters squared) $>40$ or $35 \mathrm{~kg} / \mathrm{m}^{2}$ with serious associated disorders including hypertension, type II diabetes mellitus, cardiovascular disease, sleep apnea, respiratory insufficiency, arthritis, cancer, and psychiatric disorders. Several studies have consistently shown that both, overweight and obesity, are important risk factors for the development of various functional digestive disorders, including faecal incontinence (FI) and constipation. ${ }^{1,3,4}$

Faecal incontinence is a common condition that results in recurrent uncontrolled passage of faecal material, ranging from occasional leakage of small amount of stool or passing gas to complete loss of control of bowel movements. The reported prevalence varies considerably depending on the observed population and definition or symptoms classification, ${ }^{3}$ but it can be as high as $12 \%$ in the general population. ${ }^{3}$ Although FI is well known to be more prevalent in overweight people and BMI is positively associated with pelvic floor disorders (PFDs), including urinary incontinence (UI), the prevalence of constipation in obese patients is unclear and reports are often discordant. Only one investigator has reported an association between obesity and constipation in adults, and others have studied this association in obese children. ${ }^{4,5}$ Conversely, some authors reported diarrhoea as the major symptom in the same population. 6,7

The relationship of constipation (defined as incomplete, prolonged, difficult, infrequent or painful defaecation) with lifestyle behaviours such as an unhealthy diet, smoking, excessive alcohol consumption, and lack of exercise has also been investigated. Besides, the higher intra-abdominal pressure, predisposing to acute faecal leakages or to difficult bowel emptying, might jeopardize the pelvic floor function in obese people. ${ }^{8}$

Therefore, morbid obesity seems to be associated with defaecatory disorders (DDs) such as FI and constipation. However, data on their prevalence as well as effectiveness of bariatric surgery on their correction are scant.

The aim of this study was the assessment of the effect of morbid obesity on defaecatory disorders in a cohort of patients candidate for bariatric surgery and, as a secondary end point, the evaluation of FI and constipation pattern in a subgroup of patients who already underwent sleeve gastrectomy.

\section{Patients and Methods}

A questionnaire-based study was proposed to morbidly obese, defined as having a BMI $\geq 35 \mathrm{~kg} / \mathrm{m}^{2}$ plus comorbidities or BMI $\geq 40 \mathrm{~kg} / \mathrm{m}^{2}$, who were candidates for bariatric surgery. Data included demographics, past medical, obstetric and surgical history, as well as co-morbidities.

Wexner Constipation Score (WCS) and Faecal Incontinence Severity Index (FISI) questionnaires were used to evaluate both constipation and faecal incontinence. We considered abnormal a WCS $\geq 5$ and a FISI score $\geq 10$. Identical questionnaires were completed after 3 and 6 months of follow-up. For the purpose of the study, an improvement of the WCS or FISI greater than 25\% was considered clinically relevant [expressed as mean \pm standard deviation (SD)]. For the purpose of this study, only patients who underwent sleeve gastrectomy were selected with a minimum postoperative follow-up of 6 months.

Responders who had history of chronic diarrhea, inflammatory bowel disease, major non-obstetric anal sphincter trauma, paraplegia/paresis, or previous (non-obstetric) anorectal surgery (other than uncomplicated hemorrhoidectomy) were not included in the study. Patient who underwent a different surgical procedure from the planned (including concomitant surgery for other disease) were excluded during the follow-up. Patients who had severe complications requiring longer admissions were also excluded from this study. Patients lost during the follow-up or who underwent other hospital admissions or surgeries during the follow-up were also excluded. The study was approved by the Tor Vergata University Institutional Review Board. Chi-square test and Fisher's exact test were used appropriately to compare continuous and categorical variables. $P$ value $<0.05$ was considered statistically significant.

\section{Results}

Between March 2010 and April 2011, a total of 139 candidates for bariatric surgery were enrolled in the study, 93 were females and 46 were males, with a mean age of 43 years (range 17-73). Of those, 68 underwent sleeve gastrectomy (39 females and 29 males) and fully satisfied our criteria, including a minimum follow-up of 6 months after surgery. The remaining 61 patients were excluded because of different bariatric or concomitant procedure from the initially planned at listing (45 patients), not compliant with the study after the surgery (6 patients), severe complications leading to death (1 patient) requiring longer term admissions (3 patients), lost during the follow-up (3 patients), or because they underwent anorectal or other abdominal surgery during the follow-up (3 patients).

As shown in Table 1, overall, 58.9\% of the patients reported DDs according to the above-mentioned scores. Twenty-eight patients $(20.1 \%)$ had a WCS $\geq 5$. Thirty-five patients $(25 \%)$ had a FISI $\geq 10$ while 19 patients $(13.7 \%)$ reported mixed disorders with combined abnormal scores.

BMI influences specific DDs (FI or constipation). The risk of FI increases with the BMI increase. As shown in Table 1 , about $7 \%$ of patients with BMI $<40 \mathrm{~kg} / \mathrm{m}^{2}$ had a FISI score $>10$, but this percentage raised to $40 \%$ considering patients with BMI $>50 \mathrm{~kg} / \mathrm{m}^{2}(24 \%$ when BMI ranges between 40 and $50 \mathrm{~kg} / \mathrm{m}^{2}$ ). Differently, the 
Table 1 Overall defaecatory disorders in the cohort of 139 patients listed for bariatric surgery

\begin{tabular}{lccrr}
\hline & & BMI $<40 \mathrm{~kg} / \mathrm{m}^{2}$ & $>40$ BMI $<50 \mathrm{~kg} / \mathrm{m}^{2}$ & BMI $>50 \mathrm{~kg} / \mathrm{m}^{2}$ \\
\hline No. of patients & 139 & 29 & 70 & 40 \\
FISI $>10$ (patients/\%) & $35(25.2 \%)$ & $2(6.8 \%)$ & $17(24.2 \%)$ & $13(18.5 \%)$ \\
WCS $>5$ (pts/\%) & $28(20.1 \%)$ & $10(34.4 \%)$ & $9(12.8 \%)$ & $5(12.5 \%)$ \\
WCS $>5 /$ FISI $>10$ (patients/\%) & $19(13.7 \%)$ & $7(24.1 \%)$ & $3(7.5 \%)$ \\
Patiens with DDs (patients/\%) & $82 / 139(58.9 \%)$ & $19 / 29(65.5 \%)$ & $39 / 70(55.7 \%)$ & $24 / 40(60 \%)$ \\
\hline
\end{tabular}

$B M I$ body mass index, FISI Faecal Incontinence Severity Index, WCS Wexner Constipation Score, DD defaecatory disorder

prevalence of constipation (expressed as WCS $>5$ ) and mixed disorders (expressed as WCS $>5$ and FISI $>10$ ) was more evident with BMI $<40 \mathrm{~kg} / \mathrm{m}^{2}$.

Despite overall percentage of patients with DDs were similar between patients with BMI $>50 \mathrm{~kg} / \mathrm{m}^{2}(60 \%)$ compared to patients with BMI $>40 \mathrm{~kg} / \mathrm{m}^{2}(65 \%)$, the severity of these disorders (expressed as mean FISI or WCS scores $\pm \mathrm{SD}$ ) were different according to BMI as shown in Table 2. Constipation seems to be more severe with BMI $<40 \mathrm{~kg} / \mathrm{m}^{2}$, conversely FI with BMI $>50 \mathrm{~kg} / \mathrm{m}^{2}$.

Regarding FI, this trend is even more evident and significant if we consider only the subgroup of patients with clinically relevant FI (FISI $>10$ ). Among these, mean FISI scores were $12 \pm 4$ and $18.2 \pm 6$ respectively for patients with BMI $<40$ and $>50 \mathrm{~kg} / \mathrm{m}^{2}(p=0.03)$.

Similarly and unexpectedly, although constipation seems to be more frequent with lower BMI, when we consider the subgroup with clinically relevant constipation (WCS $>5$ ), it seems to be more severe being the scores $7.1 \pm 3.5$ and $10.6 \pm$ 3.5 respectively, among patients with $\mathrm{BMI}<40$ and $>50 \mathrm{~kg} / \mathrm{m}^{2}$ $(p=0.01)$.

Considering the 68 patients who did meet our criteria and completed the study, they were 39 females and 29 males with similar BMI, respectively $47 \pm 7$ and $47.9 \pm 8.1 \mathrm{~kg} / \mathrm{m}^{2}$. Overall, $58.8 \%$ of these patients presented DDs as resumed in Table 3 . DDs distribution was similar between females and males (58.6\% vs. $59 \%$ ), with no differences according to the examined scores: FISI $>10(33.3 \%$ vs. $31 \%)$, WCS $>5$ $(17.9 \%$ vs. $10.2 \%)$ and mixed (10.2\% vs. $10.3 \%)$.

Table 2 Severity of DDs disorders expressed as mean \pm standard deviation (SD) of FISI or WCS scores among the 139 patient initially listed for surgery

\begin{tabular}{lccc}
\hline & $\begin{array}{l}\text { BMI }<40 \mathrm{~kg} / \mathrm{m}^{2} \\
29 \text { patients }\end{array}$ & $\begin{array}{l}>40 \mathrm{BMI}<50 \mathrm{~kg} / \\
\mathrm{m}^{2} 70 \text { patients }\end{array}$ & $\begin{array}{l}\mathrm{BMI}>50 \mathrm{~kg} / \mathrm{m}^{2} \\
40 \text { patients }\end{array}$ \\
\hline $\begin{array}{c}\mathrm{WCS} \\
(\text { mean } \pm \text { SD) }\end{array}$ & $5.4 \pm 4.4$ & $3.7 \pm 3.5$ & $3.8 \pm 4$ \\
$\begin{array}{c}\text { FISI } \\
(\text { mean } \pm \text { SD) }\end{array}$ & $7.8 \pm 6.5$ & $8.8 \pm 8.2$ & $10.6 \pm 8.7$ \\
\hline
\end{tabular}

$B M I$ body mass index, FISI Faecal Incontinence Severity Index, WCS Wexner Constipation Score, $S D$ standard deviation
Mean preoperative WCS was $4.1 \pm 4$ (range $0-17$ ), while the mean FISI score was $9.5 \pm 9$ (range 0-38), with no differences between females and males (WCS, $4.5 \pm 4$ vs. 3 \pm 2 ; FISI, $9.3 \pm 9$ vs. $11 \pm 8$ ). Similar to the data observed for all 139 patients listed for surgery, FI was more severe with BMI $>50 \mathrm{~kg} / \mathrm{m}^{2}$ compared to lower BMI $<40 \mathrm{~kg} / \mathrm{m}^{2}(10.3 \pm$ 9.8 vs. $2.4 \pm 5.6$, respectively). Conversely, constipation was more evident with lower BMI (3.8 \pm 4.1 vs. $0.7 \pm 2)$.

The mean BMI decreased significantly from $47 \pm 7$ to $36 \pm 6$ and to $29 \pm 4 \mathrm{~kg} / \mathrm{m}^{2}$, respectively at 3 and 6 months after surgery ( $p<0.01$ and $p<0.0001$, respectively). Mean BMI decrease was similar between females and males both at $3\left(36.2 \pm 6\right.$ and $37.2 \pm 7 \mathrm{~kg} / \mathrm{m}^{2}$, respectively) and 6 months $\left(29.2 \pm 4\right.$ and $30 \pm 6 \mathrm{~kg} / \mathrm{m}^{2}$, respectively). As shown in Table 3, improvements on DDs were already evident 3 months after surgery and were amplified thereafter at 6 months.

In particular, after 6 months, overall DDs rates were significantly reduced from $58.8 \%$ (40 patients out of 68 ) to $13 \%$ (9 out of 68). Particularly, this decrease was more evident for FI alone (expressed as percentage of patients with FISI $>10$; from $32 \%$ to $3 \%$ ) compared to constipation alone (expressed as percentage of patients with WCS $>5$; from $16 \%$ to $10 \%$ ). Similar significant decrease occurred for mixed disorders (constipation/FI). These percentages and the decrease according to the BMI decrease during the follow-up were similar between genders.

Considering the FISI score, it decreased from $10 \pm$ 8 (expressed as mean $\pm \mathrm{SD}$ ) to $3 \pm 4$ and to $1 \pm 2$ respectively at 3 and 6 months $(p=0.0001)$. Similarly for constipation, the mean WCS decreased from $3.7 \pm 3$ to $3.1 \pm 4$ and to $1.6 \pm$ 3 respectively at 3 and 6 months $(p=0.02)$.

Similarly, no differences were observed according to the gender. In particular, for female patients, the mean FISI score decreased from $9.3 \pm 9$ to $2.8 \pm 4.7$ and $0.8 \pm 2.5$ at 3 and 6 months $(p<0.0001)$ respectively, while the mean WCS decreased from $4.5 \pm 4.2$ to $3.3 \pm 5.5$ and $1.6 \pm 3.1$ at 3 and 6 months $(p=0.0146)$, respectively. For male patients, the mean FISI score decreased from $11.3 \pm 8$ to $2.7 \pm 3.7$ and $0.8 \pm 2.1$ at 3 and 6 months $(p<0.0001)$ respectively, while the mean WCS decreased from $3 \pm 2$ to $2 \pm 2.3$ and $0.6 \pm 2.2$ at 3 and 6 months $(p=0.022)$, respectively. 
Table 3 Overall defaecatory disorders in the cohort of 68 patients who underwent sleeve gastrectomy

\begin{tabular}{|c|c|c|c|c|}
\hline & Pre-op & 3 months & 6 months & $p^{\mathrm{a}}$ \\
\hline FISI $>10$ (patients $/ \%)$ & $22 / 68(32.3 \%)$ & $7(10.3 \%)$ & $2(2.9 \%)$ & $<0.0001$ \\
\hline WCS >5 (patients/\%) & $11 / 68(16.2 \%)$ & $8(11.8 \%)$ & $7(10.3 \%)$ & $<0.006$ \\
\hline WCS $>5$ and FISI $>10$ (patients/\%) & $7 / 68(10.3 \%)$ & $5(7.3 \%)$ & 0 & $<0.01$ \\
\hline Overall patient with DDs (patients/\%) & $40 / 68(58.8 \%)$ & $15(22.1 \%)$ & $9(13.2 \%)$ & $<0.0001$ \\
\hline
\end{tabular}

FISI Faecal Incontinence Severity Index, WCS Wexner Constipation Score, DD defaecatory disorder

${ }^{\text {a }}$ Statistical analysis at 6 months

\section{Discussion}

Defaecatory disorders are usually associated with UI, pelvic organ prolapse, chronic pelvic pain and sexual dysfunction under the condition named PFDs. Although several authors have assessed the association between obesity and PFDs, especially regarding UI, data about a clear link between obesity and DDs are less certain and not clearly supported by literature. This study aims at assessing the effect of morbid obesity on DDs and to evaluate FI and constipation pattern in patients who underwent sleeve gastrectomy.

The prevalence of FI is estimated to affect up to $12 \%$ of adults, while constipation up to $32 \% .{ }^{3}$ However, data regarding the prevalence of these conditions in the obese population are scant, although morbid obesity is formally recognised to be a risk factor for the occurrence of PFDs and associated symptoms that range from FI to constipation. Some authors report an increase by half in morbidly obese patients of FI and constipation compared to the general population. ${ }^{9}$

The contribution of obesity to PFDs is probably multifactorial, related to the increased intra-abdominal pressure, diabetes, nerve conduction abnormalities and intervertebral disc herniation. ${ }^{10}$ Bariatric surgery has been proven to solve most of the co-morbidities related to morbid obesity mainly hypertension, diabetes and metabolic syndrome.

Few authors described the effect of bariatric surgery and weight loss on PFDs, but studies considering DDs are rare if not absent. In this study, we evaluated the prevalence of these disorders in a cohort of patients listed for bariatric surgery. According to Emily et al., the prevalence of FI in this population is close to $30 \%$ while the constipation rate is not reported. Our study indicates that DDs occur in about $59 \%$ of the patients with no differences between females and males no differences between the patterns (FI or constipation) and apparently with similar distribution (expressed as percentage of patients with the specific disorders) according to BMI increase. However, FI seems to be more frequent with higher BMI while constipation with lower BMI. Besides, considering the severity of the specific disorders (expressed as mean FISI and WCS), the BMI increase affects negatively and significantly the scores we have used in the study.
According to other authors, central obesity may be one of the strongest modifiable independent risk factors for FI, especially in women. ${ }^{11}$ Other potential risk factors are age, parity, mode of delivery, impairments in activities of daily living and co-morbid diseases. On the other hand, constipation might be explained with a scarce dietary fiber intake. ${ }^{3}$ In our population, we did not observe differences between genders for both prevalence of DDs and severity either before or after surgery.

Burgio et al. ${ }^{12}$ described an improvement of FI symptoms in a group of patients who underwent gastric bypass (from $19.2 \%$ to $8.6 \%$ ). Wassenberg et al. ${ }^{10}$ described no significant improvement in DDs after weight loss, despite a bias might be due to the instruments they have used to evaluate colorectal symptoms.

Our study demonstrates the decrease of DDs in a homogeneous group of morbidly obese patients who underwent to sleeve gastrectomy. We selected the procedure based on two motivations: sleeve gastrectomy includes the major number of bariatric procedures in our facility and it is a pure restrictive procedure.

Similar to other malabsorpitive procedures, the gastric bypass might lead to diarrhoea secondary to micro- and macronutrients malabsorption as well as changes in intestinal bacterial flora. In our opinion, this condition would affect DDs symptoms after surgery thus the results and outcome of this study. Sleeve gastrectomy, as a restrictive procedure, does not change the physiology of the bowel thus with negligible effects on defaecation.

As expected, sleeve gastrectomy was effective in reducing BMI during the follow-up. Accordingly, overall DDs (expressed as percentage of patients with clinically relevant FISI or WCS) decreased during the follow-up. This amelioration was also reflected in the severity of the specific disorder expressed as mean FISI or WCS score. These results suggest that the reduction of intra-abdominal pressure may have supported the improvement of WS and FISI more than diabetic or metabolic syndrome resolution.

Intra-abdominal pressure influences more FI than constipation because of the strength applied on the pelvic floor and continence apparatus. As shown in this study, FI is more frequent and severe when BMI exceeds $50 \mathrm{~kg} / \mathrm{m}^{2}$ 
while constipation is more frequent with lower BMI. The fact that surgery greatly improves FI confirms the hypothesis that intra-abdominal pressure reduction is a main factor for DDs occurrence.

It can be argued that the obtained changes in eating habits after surgery might explain the observed improvements. However, it is possible that the new alimentary regimen might contribute as an additional factor, but it is also true that these patients usually experienced several periods of diet without benefits in terms of weight reduction and bowel function. ${ }^{10}$

In conclusion, our findings suggest a clear association between DDs and obesity. Our results confirm that the DDs in terms of FI and/or constipation prevalence and severity improve after sleeve gastrectomy.

\section{References}

1. Wael Agur \& Diaa E. E. Rizk. Obesity and urinary incontinence in women: is the black box becoming grayer? Int Urogynecol $J$ (2011) 22:257-258

2. Wang Y, Beyoudoun MA. The obesity epidemic in the United States-gender, age, socioeconomic, racial/ethnic, and geographic characteristics: a systematic review and meta-regression analysis. Epidemiol Rev. 2007; 29:6-28.

3. A.D.Markland, H.E. Richter, K. L. Burgio, C. Bragg, A.L. Hernandez and L.L. Subak Fecal incontinence in obese women with urinary incontinence: prevalence and role of dietary fiber intake Am J Obstet Gynecol. 2009 May; 200(5):566.el-566.e6

4. Delgado-Aros S, Locke 3rd GR, Camillieri M et al. Obesity is associated with increased risk of gastrointestinal symptoms: a population-based study. Am J Gastroenterol. 2004; 99 (9): 1801-6

5. Talley NJ, Quan C, Jones MP, et al. Association of upper and lower gastrointestinal tract symptoms with body mass index in an Australian cohort. Neurogastroenterol Motil. 2004; 16 (4):413-9

6. Talley NJ, Howell S, Poulton R. Obesity and chronic gastrointestinal tract symptoms in young adults: a birth cohort study. Am J Gastoenterol. 2004; 99 (9): 1807-14

7. Delgado-Aros S, Camillieri M, Garcia MA, Burton D, Busciglio I. High body mass alters colonic sensory-motor function and transit in humans. Am J Physiol Gastrointest Liver Physiol. 2008; 295 (2):G382-8

8. A.E. Bharucha. Incontinence: An underappreciated problem in obesity and bariatric surgery. Dig. Dis. Sci (2010) 55:24282430

9. Whitcomb EL, Lukacz ES, Lawrence JM, Nager CW, Luber KM Prevalence and degree of bother from pelvic floor disorders in obese women. Int Urogynecol J (2009) 20:289-294

10. Nir Wasserberg. Effect of Surgically Induced Weight Loss on Pelvic Floor Disorders in Morbidly Obese Women Annals of Surgery, Volume 249, Number 1, January 2009

11. Wasserberg N, Haney M, Petrone P., Crookes P., Rosca J, Ritter M, Kaufman HS. Fecal incontinence among morbid obese women seeking for weight loss surgery: an underappreciated association with adverse impact on quality of life. Int J Colorectal Dis. 2008 May;23(5):493-7. Epub 2008 Jan 29.

12. Burgio KL, Richter HE, Clements RH, Redden DT, Goode PS. Changes in urinary and fecal incontinence symptoms with weight loss surgery in morbidly obese women. Obstet Gynecol. 2007;110:1034-1040-.

\section{Discussant}

Dr. Lee L. Swanstrom (Portland, OR): Dr. Franceschilli and her colleagues present an interesting study on the prevalence and effect of bariatric surgery on the incidence of defaecatory disorders (DD) - namely constipation or faecal incontinence. Both problems were quantified using validated scoring systems although it should be mentioned that neither system was specifically validated in the morbidly obese population.

Two populations were studied: 143 patients waiting for bariatric surgery and 44 patients who had bariatric surgery and had follow-up questionnaires to determine the effect on the surgery on their problem. They found, as have many others, that DD is very prevalent in the morbidly obese $(61 \%$ in their population with one third constipated and about one third with faecal incontinence). The second group showed little effect on constipation but did show improvement in faecal incontinence; a change which correlated with the amount of weight lost. The report is weakened by the fact that we are not given a clue as to why the patients of either group had DD before surgery-how many had diarrhea? Both to start with and after surgery.

How valid is the FISI score for the morbidly obese? Is it possible that their complaints of "faecal soilage" are more related to mechanical and ergonomic issues than to a true DD?

The authors declare that faecal incontinence is progressively improved with weight loss - but is it not equally possible that the improvement seen is due to a change in their eating habits and that better eating leads both to weight loss and to better bowel function?

In light of their results, I might suggest to the authors that a better title for their report is "Fecal incontinence is improved following gastric sleeve resection."

Thank you.

\section{Closing Discussant}

Dr. Pierapolo Sileri: Prof. Swanstrom, thank you for your questions and comment.

Regarding the first question, in our study, we did not include patients with history of chronic diarrhea, inflammatory bowel disease, major non-obstetric anal sphincter trauma, or previous anorectal surgery (other than uncomplicated hemorrhoidectomy).

Fecal Incontinence Severity Index ${ }^{[6]}$ is based on a typeby-frequency matrix with four types of leakage (gas, mucus, liquid stool, and solid stool) and five frequencies (one to three times per month, once per week, twice per week, once per day, twice or more per day). We believe that faecal incontinence is related to the increased intra- 
abdominal pressure, diabetes, nerve conduction damage and generalized weakness and sagging of the pelvic floor. In our study, FI progressively improved with weight loss but we agree that the improvement seen might be also due to a change in eating habits and a better eating.

This study aims at assessing the effect of morbid obesity on DDs and to evaluate FI and constipation pattern in patients who underwent sleeve gastrectomy thus excluding other bariatric procedures such as gastric bypass which may lead to bowel symptoms as diarrhoea due to malabsorption of micro- and macronutrients and changes in bacterial flora of the bowel. Sleeve gastrectomy counts the major number of bariatric procedure in our facility and, as a restrictive procedure, does not change the physiology of the bowel thus with neglectable effects on defaecation.

Thank you. 